Elaine Cristina Marqueze ${ }^{1,2}$

Marcelo Just da Silva²

Claudia Roberta de Castro Moreno

${ }^{1}$ Departamento de Saúde Ambiental da Faculdade de Saúde Pública da Universidade de São Paulo - USP

${ }^{2}$ Universidade do Extremo Sul Catarinense - UNESC

* Trabalho apresentado no XI Congresso Brasileiro de Sono, em Fortaleza/CE, de 11 a 14/11/07.

Contato:

Claudia Roberta de Castro Moreno Av. Dr. Arnaldo, 715 - São Paulo, SP CEP 01246-904

E-mail:

crmoreno@usp.br

\section{Qualidade de sono, atividade física durante o tempo de lazer e esforço físico no trabalho entre trabalha- dores noturnos de uma indústria cerâmica*}

\author{
Sleep quality, physical activity during leisure time and physical \\ effort at work among night workers of a ceramic industry
}

\section{Resumo}

Este estudo teve o objetivo de verificar a relação entre qualidade do sono e atividade física durante o tempo de lazer entre trabalhadores noturnos com um elevado esforço físico no trabalho (média de 14.245; DP $3.456 \mathrm{kcal} / \mathrm{semana}$ ). Também foi avaliada a relação entre a qualidade do sono e o nível do esforço físico no trabalho. Um total de 19 trabalhadores noturnos de uma indústria cerâmica respondeu o índice da qualidade do sono de Pittsburgh (PSQI) e o questionário internacional da atividade física (IPAQ). Foi calculado o dispêndio calórico total por semana para cada trabalhador através do equivalente metabólico (METs). Os trabalhadores foram distribuídos em dois grupos, de acordo com os escores do PSQI. Foi realizada a análise descritiva dos dados por meio de médias, valores mínimo e máximo, proporções e desvios-padrão. A relação entre qualidade do sono e atividade física foi avaliada pelo teste Mann-Whitney. O grupo de qualidade do sono ruim mostrou nível mais elevado de gasto energético no trabalho que o outro grupo, o que sugere uma relação entre gasto energético no trabalho e qualidade de sono. Distúrbios do sono e sonolência diurna também contribuíram para a qualidade de sono ruim. Esses resultados, embora preliminares, revelam uma tendência de que o elevado esforço físico no trabalho noturno contribui para baixa qualidade de sono.

Palavras-chave: qualidade do sono, atividade física, trabalhadores noturnos.

\begin{abstract}
This study aimed at verifying the relationship between sleep quality and physical activity during leisure time among night workers with high physical effort at work (average 14,245; SD 3,456 kcal/week). Also, the relationship between sleep quality and level of physical effort at work was evaluated. A total of 19 night workers of a ceramic industry filled in the Pittsburgh Sleep Quality Index (PSQI) and the International Physical Activity Questionnaire (IPAQ). The total energy expenditure per week for each worker was expressed by the metabolic equivalent (METS). Workers were distributed in two groups, according to the PSQI scores. We performed the descriptive analysis of data, calculating average, minimum and maximum values, percentages and standard deviations. The relationship between quality of sleep and physical activity was assessed by Mann-Whitney Test. The poor sleep quality group showed higher level of energy expenditure at work than the other group, suggesting a relationship between energy expenditure at work and sleep quality. Sleep disorders and diurnal sleepiness contributed to the poor sleep quality as well. Although these are preliminary results, they indicate that higher physical effort exerted at night work contributes to low sleep quality.
\end{abstract}

Keywords: sleep quality, physical activity, night workers. 


\section{Introdução}

A crescente demanda por trabalhadores do turno noturno vem atender os países industrializados que necessitam de produção e serviços ininterruptamente. Como consequência, trabalhadores invertem o dia pela noite, alterando, assim, seu ciclo vigília-sono. A inversão dos horários de dormir e acordar leva à privação de sono, uma vez que trabalhadores do turno noturno tendem a dormir cerca de duas horas a menos que os do turno diurno (COREN, 1996).

Além de efeitos nocivos à saúde, Reilly e Edwards (2007) afirmam que, quando há privação de sono, episódios de comportamento bizarros ou alucinações são frequentemente percebidas. O trabalho noturno também tem sido com frequência associado a distúrbios de sono (AKERSTEDT et al., 2002). Na população em geral, a prevalência desses distúrbios está entre 35\% e 41\% (SHERRILL; KOTCHOU; QUAN, 1998).

Mesmo considerando a alta prevalência de distúrbios do sono e seus impactos negativos, o índice de pessoas que recebem tratamento ainda é baixo (MONTGOMERY; DENNIS, 2004). Os tratamentos comumente utilizados são os farmacológicos, embora sua utilização possa trazer complicações, tanto na tolerância, como na dependência (MONTGOMERY; DENNIS, 2004; PASSOS et al., 2007). Já os tratamentos não farmacológicos são indicados por não apresentarem risco de tolerância nem de dependência e também por terem um maior tempo de ação (MONTGOMERY; DENNIS, 2004). Apesar dos tratamentos não farmacológicos mostraremse efetivos, a falta de conhecimento e o acesso limitado a esses tratamentos são apontados como os principais motivos para sua não utilização (MONTGOMERY; DENNIS, 2004; PASSOS et al., 2007). Dentre os mais estudados estão higiene do sono, relaxamento muscular, restrição do sono, controle de estímulos, terapia cognitiva, fototerapia e exercício físico (MONTGOMERY; DENNIS, 2004; PASSOS et al., 2007). Especificamente no caso do exercício físico, diversos pesquisadores e organizações de saúde têm recomendado sua prática (LI et al., 2004).

Diversos estudos mostram que o exercício físico está associado ao aumento da duração e da qualidade de sono, bem como à diminuição da latência, da sonolência diurna, dos despertares noturnos, dos distúrbios do sono, do uso de medicamentos ou de álcool (SINGH; CLEMENTS; FIATARONE, 1997; SHERRILL; KOTCHOU; QUAN, 1998; TANAKA et al., 2002; BENLOUCIF et al., 2004; LI et al., 2004; ATLANTIS et al., 2006; GUIMARÃES et al., 2008).

Até maio de 2008, havia no Pubmed 6.732 artigos com a palavra-chave sleep quality (qualidade do sono). Na mesma época, foram encontrados 309 artigos, após a realização de um refinamento com a palavra-chave exercise (exercício). Em outra busca bibliográfica no Pubmed com o descritor Pittsburgh sleep quality índex (Índice de Qualidade de Sono de Pittsburgh - PSQI), que é um instrumento de avaliação da qualidade de sono comumente utilizado, foram encontrados 387 artigos. Neste caso, foi também realizado um refinamento da busca bibliográfica com a palavra-chave exercise, o qual resultou em 14 artigos. Destes 323 artigos (309 da primeira busca e 14 da segunda), apenas 16 eram estudos de campo e/ou estudavam a relação entre qualidade de sono e exercício físico em pessoas com ou sem distúrbios de sono (Quadro 1).

Esses estudos evidenciam que os principais benefícios observados no sono, decorrentes da prática de exercícios físicos, foram: melhora da qualidade do sono, diminuição do tempo de latência e da prevalência de distúrbios do sono, aumento na duração do sono e menor frequência de microdespertares (SHAPIRO; BACHMAYER, 1988; KING et al., 1997; SINGH; CLEMENTS; FIATARONE, 1997; SHERRILL; KOTCHOU; QUAN, 1998; BENLOUCIF et al., 2004; ATLANTIS et al., 2006; GUIMARÃES et al., 2008).

Outros autores, como Elavsky e McAuley (2007) e Imaki et al. (2002), não encontraram melhoras significativas na qualidade de sono relacionadas à prática de exercícios físicos. Além destes, Vuori et al. (1988) afirmam que o exercício físico vigoroso tem um efeito negativo sobre o sono. Esses autores observaram que somente exercícios físicos de intensidade leve ou moderada provocam impacto positivo na qualidade do sono. Geroldi et al. (1996) mostraram ainda que pessoas com histórico de vida profissional com baixo esforço físico apresentaram melhor qualidade de sono em comparação aos que tiveram trabalho com um esforço físico elevado. Em resumo, parece lógico supor que o esforço físico tenha uma influência na qualidade do sono, seja positiva ou não.

Nesse contexto, o objetivo deste estudo foi verificar a relação entre qualidade do sono e a atividade física no lazer entre trabalhadores noturnos com um elevado esforço físico no trabalho. Também foi avaliada a relação entre a qualidade do sono e o nível do esforço físico no trabalho.

\section{Metodologia}

\section{População}

Este estudo foi realizado com 19 trabalhadores do turno noturno fixo (21h às $05 \mathrm{~h})$ de uma indústria cerâmica. Foram incluídos na amostra os trabalhadores com mais de seis meses na empresa e que aceitaram participar voluntariamente da pesquisa.

Todos eram do sexo masculino, com idade média de 36,3 anos (DP 7,4 anos), trabalhavam na empresa em média há 7,1 anos (DP 4,7 anos) e no turno noturno em média há 6,5 anos (DP 4,2 anos). A jornada de trabalho semanal era de 48 horas e $42,1 \%$ realizavam horas extras (média de 13 horas por semana).

Os setores de trabalho dos participantes da pesquisa eram: produção (79\%), mecânica (10,5\%) e operação de 
Quadro 1 Estudos de campo sobre qualidade de sono e exercício físico.

\begin{tabular}{|c|c|c|c|}
\hline Autores & $\begin{array}{l}\text { Ano de publi- } \\
\text { cação }\end{array}$ & População de estudo & Tipo de estudo \\
\hline Guimarães et al. & 2008 & $\begin{array}{l}53 \text { idosas ativas e } 48 \text { sedentárias ( } 60 \\
\text { anos ou mais) }\end{array}$ & Transversal \\
\hline Elavsky e McAuley & 2007 & $\begin{array}{l}164 \text { mulheres (idade média } 49,9 \\
\text { anos) }\end{array}$ & $\begin{array}{l}\text { Experimental - } 16 \text { semanas (caminhada ou } \\
\text { yoga) }\end{array}$ \\
\hline Atlantis et al. & 2006 & 73 trabalhadores & $\begin{array}{l}\text { Experimental - } 24 \text { semanas (exercícios } \\
\text { físicos no ambiente de trabalho) }\end{array}$ \\
\hline Gambelunghe et al. & 2005 & 18 ratos adultos & Experimental (caminhada ou corrida) \\
\hline Mori et al. & 2005 & 777 trabalhadores aposentados & Transversal \\
\hline Benloucif et al. & 2004 & $\begin{array}{l}12 \text { idosos ( } \text { média de idade }=74,6 \\
\text { anos) }\end{array}$ & $\begin{array}{l}\text { Experimental - } 14 \text { dias (alongamento, } \\
\text { aeróbico de baixo impacto e jogos recrea- } \\
\text { tivos) }\end{array}$ \\
\hline Li et al. & 2004 & $\begin{array}{l}118 \text { idosos (idade média }=75,30 \\
\text { anos participantes do Tai Chi } / \text { idade } \\
\text { média participantes exercícios de } \\
\text { baixo impacto }=75,45 \text { anos) }\end{array}$ & $\begin{array}{l}\text { Experimental - } 24 \text { semanas (Tai Chi e Exer- } \\
\text { cício de baixo impacto) }\end{array}$ \\
\hline Tanaka et al. & 2002 & 11 idosos (idade média = 73,8 anos) & $\begin{array}{l}\text { Experimental - } 4 \text { semanas (exercício físico + } \\
\text { cochilo depois do almoço) }\end{array}$ \\
\hline Imaki et al. & 2002 & $\begin{array}{l}1.652 \text { a } 1.860 \text { trabalhadores (idade } \\
\text { entre } 20 \text { a } 59 \text { anos) }\end{array}$ & Longitudinal (1992 a 1998) \\
\hline Sherril et al. & 1998 & $\begin{array}{l}722 \text { adultos ( } 319 \text { homens - idade } \\
\text { média }=54,1 \text { anos e } 403 \text { mulheres } \\
\text { - idade média }=59,9 \text { anos) }\end{array}$ & Transversal \\
\hline Singh et al. & 1997 & 32 idosos (60 a 84 anos) & $\begin{array}{l}\text { Experimental - } 10 \text { semanas (exercícios } \\
\text { com peso) }\end{array}$ \\
\hline King et al. & 1997 & $\begin{array}{l}29 \text { mulheres e } 14 \text { homens (idades en- } \\
\text { tre } 50 \text { a } 76 \text { anos), que apresentavam } \\
\text { queixas moderadas de sono }\end{array}$ & $\begin{array}{l}\text { Experimental - } 16 \text { semanas (exercícios } \\
\text { aeróbios moderados) }\end{array}$ \\
\hline Geroldi et al. & 1996 & 449 idosos (75 anos ou mais) & Transversal \\
\hline Weydahl & 1991 & 465 estudantes do $2^{\circ}$ grau & Transversal \\
\hline Vuori et al. & 1988 & $\begin{array}{l}1.200 \text { adultos (idade entre } 36 \text { a } 50 \\
\text { anos) }\end{array}$ & Transversal \\
\hline Shapiro e Bachmayer & 1988 & 800 adultos & Transversal \\
\hline
\end{tabular}

máquinas $(10,5 \%)$. A maioria não possuía outro emprego $(94,7 \%)$ e era casada $(73,7 \%)$.

A coleta de dados foi realizada após aprovação do Comitê de Ética da Universidade do Extremo Sul Catarinense (UNESC), de acordo com as normas da Resolução 196/96 do Conselho Nacional de Saúde.

\section{Instrumentos para coleta de dados}

Os trabalhadores responderam dois questionários: Índice de Qualidade de Sono de Pittsburgh (PSQI) e o Questionário Internacional de Atividade Física (IPAQ) - versão longa.

O PSQI é um questionário com 19 questões que avalia as características do sono durante o intervalo de um mês. Dezenove itens individuais são agrupados em sete componentes, cada um avaliado em uma escala de 0 a
3 com mesmo peso. A soma dos valores desses componentes constitui o índice PSQI global, que varia entre 0 a 21 pontos. Quando o PSQI global atinge valor igual ou superior a cinco, o avaliado é classificado como portador de sono ruim (BUYSSE et al., 1989).

O IPAQ é um questionário validado no Brasil e é composto por cinco seções: atividade física no trabalho; atividade física como meio de transporte; atividade física em casa, trabalho, tarefas domésticas e cuidar da família; atividades físicas de recreação, esporte, exercício e de lazer; tempo gasto sentado (BARROS; NAHAS, 2003). Cada seção é relacionada ao tempo que o indivíduo gasta fazendo atividade física em uma semana habitual. O equivalente metabólico foi calculado pelo tempo total despendido em cada atividade registrada no IPAQ (AINSWORTH et al., 2000). Para cálculo do dispêndio energético na unidade de Kcal/ min, a fórmula $0,0175 \mathrm{kcal} / \mathrm{kg} / \mathrm{min}$ x MET x massa cor- 
poral foi utilizada, na qual $0,0175 \mathrm{kcal} / \mathrm{kg} / \mathrm{min}$ é equivalente a 1 MET.

Para o cálculo do Índice de Massa Corporal (IMC), foram medidas a massa corporal e a estatura dos trabalhadores. O cálculo e a classificação do IMC seguiram o proposto pela Organização Mundial da Saúde (WORLD HEALTH ORGANIZATION, 1997).

\section{Análise dos dados}

Foi realizada a análise descritiva dos dados por meio de médias, valores mínimo e máximo, proporções e desvios-padrão. De acordo com os escores do PSQI, os trabalhadores foram distribuídos em dois grupos: um incluindo os que reportaram qualidade de sono boa e outro incluindo os que reportaram qualidade de sono ruim. A relação entre qualidade do sono e atividade física foi avaliada pelo teste Mann-Whitney.

\section{Resultados}

A partir da medição da massa corporal e da estatura, calculou-se o Índice de Massa Corporal e constatou-se que onze trabalhadores (58\%) apresentaram IMC acima de $25 \mathrm{~kg} / \mathrm{m}^{2}$; entre esses, $45 \%$ foram classificados como 'sobrepeso' e 55\% 'obesos grau I'.

A Tabela 1 apresenta a média do gasto energético em cada situação diária identificada pelo IPAQ.
Observou-se que os trabalhadores possuem uma atividade laboral muito intensa fisicamente, o que representa uma média de gasto energético diário de 2.374 kcal. Vale destacar que o gasto energético diário no trabalho variou entre 2.822 e 7.938 kcal. Outro dado que se destaca é o gasto energético no lazer, sendo o menor gasto energético em relação a todas as seções pesquisadas.

O índice de qualidade do sono de Pittsburgh (PSQI) é composto por sete itens, os quais são chamados de componentes. A Tabela 2 apresenta a descrição desses componentes e os respectivos resultados encontrados.

A partir da soma dos sete componentes do PSQI, calculou-se a qualidade do sono dos pesquisados. Os dados mostraram que $21 \%$ dos trabalhadores possuíam qualidade subjetiva do sono 'boa' e 79\% qualidade subjetiva do sono 'ruim'.

A partir da observação de características dos trabalhadores dos dois grupos (boa qualidade de sono e qualidade de sono ruim), notou-se maior gasto energético na atividade física realizada no trabalho, na atividade física habitual total e no escore global do PSQI entre os trabalhadores do grupo de má qualidade do sono. Os resultados da análise mostraram não haver diferenças entre os grupos em relação à idade, IMC, tempo no trabalho noturno e gasto energético no lazer (Tabela 3).

Os componentes do PSQI que mais contribuíram para a qualidade de sono ruim foram os distúrbios do sono e a sonolência diurna (Tabela 4).

Tabela 1 Média do gasto energético da situação habitual observada por meio do Questionário Internacional de Atividade Física, por semana.

\begin{tabular}{lcc}
\hline \multicolumn{1}{c}{ Atividades } & Gasto energético médio / semana (Kcal) & $D P($ Kcal) \\
\hline Trabalho & 14.245 & 3.456 \\
Transporte & 2.665 & 1.661 \\
Domésticas & 2.335 & 2.035 \\
Lazer & 1.495 & 1.776 \\
Sentado & 2.206 & 1.605 \\
\hline Kcal total & 22.947 & 5.936 \\
\hline
\end{tabular}


Tabela 2 Frequência absoluta e relativa dos componentes do Índice de Qualidade de Sono de Pittsburgh.

\begin{tabular}{|c|c|c|}
\hline COMPONENTES & $n$ & $\%$ \\
\hline \multicolumn{3}{|l|}{ 1. Qualidade subjetiva do sono } \\
\hline Muito boa & - & - \\
\hline Boa & 14 & 73,7 \\
\hline Ruim & 4 & 21,0 \\
\hline Muito ruim & 1 & 5,3 \\
\hline \multicolumn{3}{|l|}{ 2. Latência do sono } \\
\hline \multicolumn{3}{|l|}{ Tempo para pegar no sono } \\
\hline$<\mathrm{ou}=15$ minutos & 10 & 52,7 \\
\hline $16-30$ minutos & 9 & 47,3 \\
\hline 31 - 60 minutos & - & - \\
\hline$>60$ minutos & - & - \\
\hline \multicolumn{3}{|c|}{$\begin{array}{l}\text { Demorou mais de } 30 \text { minutos para pegar no sono } \\
\text { no mês passado }\end{array}$} \\
\hline Nenhuma vez & 8 & 42,2 \\
\hline Menos de uma vez por semana & 6 & 31,5 \\
\hline Uma ou duas vezes por semana & 4 & 21,0 \\
\hline Três vezes ou mais por semana & 1 & 5,3 \\
\hline \multicolumn{3}{|l|}{ 3. Duração do sono (horas) } \\
\hline$>7$ horas & 10 & 52,7 \\
\hline 6 a 7 horas & 7 & 36,8 \\
\hline 5 a 6 horas & 2 & 10,5 \\
\hline$<5$ horas & - & - \\
\hline \multicolumn{3}{|l|}{ 4. Eficiência do sono } \\
\hline$>85 \%$ & 18 & 94,7 \\
\hline $75 \%$ a $84 \%$ & 1 & 5,3 \\
\hline $65 \%$ a $74 \%$ & - & - \\
\hline$<65 \%$ & - & - \\
\hline \multicolumn{3}{|l|}{ 5. Distúrbios do sono } \\
\hline Nenhuma vez por semana & - & - \\
\hline Menos de 1 vez por semana & 10 & 52,6 \\
\hline 1 a 2 vezes por semana & 7 & 36,8 \\
\hline 3 vezes ou mais por semana & 2 & 10,6 \\
\hline \multicolumn{3}{|l|}{ 6. Uso de medicamentos para dormir } \\
\hline Nenhuma vez por semana & 18 & 94,7 \\
\hline Menos de 1 vez por semana & - & - \\
\hline 1 a 2 vezes por semana & 1 & 5,3 \\
\hline 3 vezes ou mais por semana & - & - \\
\hline \multicolumn{3}{|c|}{ 7. Sonolência diurna } \\
\hline \multicolumn{3}{|c|}{ Dificuldade de se manter acordado no mês passado } \\
\hline Nenhuma vez na semana & 12 & 62,9 \\
\hline Menos de 1 vez por semana & 5 & 26,5 \\
\hline De 1 a 2 vezes por semana & 1 & 5,3 \\
\hline De 3 vezes ou mais por semana & 1 & 5,3 \\
\hline \multicolumn{3}{|c|}{$\begin{array}{l}\text { Indisposição ou falta de entusiasmo para realizar as } \\
\text { atividades diárias }\end{array}$} \\
\hline Nenhuma indisposição & 2 & 10,5 \\
\hline Pequena indisposição & 5 & 26,5 \\
\hline Moderada indisposição & 9 & 47,3 \\
\hline Muita indisposição & 3 & 15,7 \\
\hline Total & 19 & 100 \\
\hline
\end{tabular}


Tabela 3 Características dos trabalhadores de acordo com os dois grupos de qualidade de sono, comparadas pelo teste U de Mann-Whitney.

\begin{tabular}{lccc}
\hline \multicolumn{1}{c}{ Características dos trabalhadores } & $\begin{array}{c}\text { Qualidade do } \\
\text { sono boa } \\
(n=4) \\
\text { Média }(D P)\end{array}$ & $\begin{array}{c}\text { Qualidade do sono } \\
\text { ruim } \\
(n=15) \\
\text { Média }(D P)\end{array}$ & $p$ \\
\hline Idade (anos) & $38,75(5,06)$ & $35,53(8,18)$ & 0,424 \\
IMC $\left(\mathrm{kg} / \mathrm{m}^{2}\right)$ & $25,25(1,89)$ & $27,33(4,20)$ & 0,423 \\
Tempo no trabalho noturno (anos) & $6,75(3,20)$ & $6,20(4,49)$ & 0,548 \\
Gasto energético despendido no lazer $(\mathrm{kcal})$ & $1,444(2.147)$ & $1,508(1.751)$ & 0,762 \\
Gasto energético despendido no trabalho $(\mathrm{kcal})$ & $10,779(947)$ & $15,169(3.289)$ & $0,016^{*}$ \\
Dispêndio energético total (kcal) & $18,688(3.881)$ & $24,083(5.959)$ & $0,045^{*}$ \\
Índice de Qualidade do Sono de Pittsburgh & $3,25(0,50)$ & $6,67(2,04)$ & $0,002^{*}$ \\
\hline
\end{tabular}

"p significativo $(p<0,05)$

Tabela 4 Componentes do Índice de Qualidade de Sono de Pittsburgh de acordo com os dois grupos de qualidade de sono.

\begin{tabular}{|c|c|c|}
\hline Componentes do PSQI & $\begin{array}{c}\text { Qualidade de sono } \\
\text { boa } \\
(n=4)\end{array}$ & $\begin{array}{c}\text { Qualidade de sono } \\
\text { ruim } \\
(n=15)\end{array}$ \\
\hline \multicolumn{3}{|l|}{ Qualidade do sono } \\
\hline Muito boa / Boa & 4 & 14 \\
\hline Ruim / Muito ruim & - & 1 \\
\hline \multicolumn{3}{|l|}{ Latência do sono } \\
\hline$<=15 \mathrm{~min} / 16-30 \mathrm{~min}$ & 4 & 12 \\
\hline $31-60 \mathrm{~min} />60 \mathrm{~min}$ & - & 3 \\
\hline \multicolumn{3}{|l|}{ Duração do sono } \\
\hline$>7 \mathrm{~h} / 6-7 \mathrm{~h}$ & 4 & 13 \\
\hline $5-6 h /<5 h$ & - & 2 \\
\hline \multicolumn{3}{|l|}{ Eficiência do sono } \\
\hline$>85 \% / 75 \%-84 \%$ & 4 & 15 \\
\hline $65 \%-74 \% /<65 \%$ & - & 0 \\
\hline \multicolumn{3}{|l|}{ Distúrbios do sono } \\
\hline Nenhum / Menos de 1 vez/semana & 4 & 6 \\
\hline 1 a 2 vezes/semana / 3 ou mais vezes/semana & - & 9 \\
\hline \multicolumn{3}{|l|}{ Uso de medicamentos para dormir } \\
\hline Nenhum / Menos de 1 vez/semana & 4 & 14 \\
\hline 1 a 2 vezes/semana / 3 ou mais vezes/semana & - & 1 \\
\hline \multicolumn{3}{|l|}{ Sonolência diurna } \\
\hline Nenhuma / Pequena & 4 & 7 \\
\hline Moderada / Muita & - & 8 \\
\hline
\end{tabular}




\section{Discussão}

Os resultados deste estudo sugerem que o índice de qualidade do sono varia de acordo com o gasto energético médio no trabalho e nas atividades habituais totais, ou seja, revelam uma tendência de que maior gasto energético contribua para piores índices de qualidade de sono. Essa tendência corrobora o pressuposto de que um elevado gasto energético no trabalho prejudica a qualidade de sono (VUORI et al., 1988; GEROLDI et al., 1996).

O esforço físico dos trabalhadores estudados foi muito elevado no trabalho, uma vez que sua atividade é de alta demanda física. No entanto, no lazer, momento no qual o exercício físico é recomendado para se alcançar bons níveis de condicionamento e consequente melhora da saúde, os pesquisados apresentaram valores inferiores ao recomendado, que é de $2.000 \mathrm{Kcal} / \mathrm{semana}$ (NAHAS, 2003). Vale ressaltar que este gasto energético foi o mais baixo em comparação com as demais atividades pesquisadas pelo IPAQ. Este fato talvez ocorra por cansaço excessivo decorrente do trabalho, bem como pela realização do trabalho noturno. Reilly e Edwards (2007) destacam que os trabalhadores em turnos frequentemente possuem dificuldades em organizar suas atividades domésticas e atléticas.

Outro dado relevante é sobre a duração do exercício físico no lazer e sua relação com a melhora do sono. Sherrill, Kotchou e Quan (1998) encontraram, em sua meta-análise sobre o tema qualidade de sono e exercício físico, que o efeito do exercício físico no sono é observado somente entre os que o praticam com mais de uma hora de duração. Shapiro e Bachmayer, já em

\section{Referências}

AINSWORTH, B. E. et al. Compendium of physical activities: an update of activity codes and MET intensities. Medicine and Science in Sports and Exercise, United States, n. 32, p. S498-516, Sept. 2000. Supplement 9.

AKERSTEDT, T. et al. Sleep disturbances, work stress and work hours, a cross-sectional study. Journal of Psychosomatic Research. England, v. 53, n. 3, p. 741748, Sept. 2002.

ATLANTIS, E. et al. Worksite intervention effects on sleep quality: a randomized controlled trial. Journal of Occupational Health Psychology, United States, v. 11, n. 4, p. 291-304, Oct. 2006.

BARROS, M. V. G.; NAHAS, M. V. Medidas da atividade física; teoria e aplicação em diversos grupos populacionais. Londrina: Midiograf, 2003.

BENLOUCIF, S. et al. Morning or evening activity improves neuropsychological performance and
1988, publicaram os dados de um estudo transversal realizado com 800 adultos, em que verificaram que o exercício físico praticado pelo menos duas vezes por mês já é fator de proteção para o sono (SHAPIRO; BACHMAYER, 1988).

A não realização de exercício físico no lazer também pode implicar em outros problemas, como, por exemplo, distúrbios músculo-esqueléticos, posto que o trabalho dos pesquisados é essencialmente físico o que exige um bom condicionamento, situação essa provavelmente não presente e corroborada pelo elevado número de trabalhadores classificados como 'sobrepesos' e 'obesos'.

Verificou-se que, dos componentes do PSQI, os distúrbios do sono e a sonolência diurna foram os que mais contribuíram para uma qualidade do sono ruim. Esses dados se assemelham aos encontrados por Konrad (2005) e Osório et al. (2002), que também encontraram esses mesmos componentes influenciando a qualidade do sono.

É importante ressaltar que o tamanho da amostra de trabalhadores estudados e o uso de instrumentos subjetivos, tanto para a qualidade de sono, quanto para o gasto energético, devem ser considerados uma limitação deste estudo.

A má qualidade de sono parece estar relacionada ao maior gasto energético realizado no trabalho. Em outras palavras, os resultados deste estudo, embora preliminares, sugerem a necessidade de se avaliar o gasto energético no trabalho em estudos sobre a qualidade do sono de trabalhadores.

subjective sleep quality in older adults. Sleep, United States, v. 27, n. 8, p. 1542-1551, Dec. 2004.

BUYSSE, D. J. et al. The Pittsburgh sleep quality index: a new instrument for psychiatric practice and research. Psychiatry Research, Ireland, v. 28, n. 2, p. 193-213, May. 1989.

COREN, S. Sleep thieves: an eye-opening exploration into the science and mysteries of sleep. New York: The Free Press, 1996.

ELAVSKY, S.; MCAULEY, E. Lack of perceived sleep improvement after 4-month structured exercise programs. Menopause, United States, v. 14, n. 3, p. 535-540, May/June 2007. Part 1.

GAMBELUNGHE, C. et al. Effects of light physical exercise on sleep in middle-aged rats. International Journal of Sports Medicine, v. 26, n. 5, p. 327-331, June 2005. 
GEROLDI, C. et al. Principal lifetime occupation and sleep quality in the elderly. Gerontology, Switzerland, v. 42, n. 3, p. 163-169, 1996.

GUIMARÃES, L. H. C. T. et al. Physically active elderly women sleep more and better than sedentary women. Sleep Medicine, Netherland, v. 9, n. 5, p. 488-493, July 2008.

IMAKI, M. et al. An Epidemiological study on relationship between the hours of sleep and life style factors in Japanese factory workers. Journal of Physiological Anthropology and Applied Human Science, Japan, v. 21, n. 2, p. 115-120, 2002.

KING, A. C. et al. Moderate-intensity exercise and self-rated quality of sleep in older adults. A randomized controlled trial. The Journal of the American Medical Association, United States, v. 277, n. 1, p. 32-37, Jan. 1997.

KONRAD, L. M. Efeito agudo do exercício físico sobre a qualidade de vida de mulheres com síndrome da fibromialgia. 2005. 129 f. Dissertação (Mestrado em Educação Física)-Universidade Federal de Santa Catarina, Florianópolis, 2005.

LI, F. et al. Tai chi and self-rated quality of sleep and daytime sleepiness in older adults: a randomized controlled trial. Journal of the American Geriatrius Society, United States, v. 52, n. 6, p. 892-900, June 2004.

MONTGOMERY, P.; DENNIS, J. A systematic review of non-pharmacological therapies for sleep problems in later life. Sleep Medicine Reviews, England, v. 8, n. 1, Feb. p. 47-62, 2004.

MORI, M. et al. Effects of shift work on quality of sleep among retired workers. Sangyo Eiseigaku Zasshi, Japan, v. 47, n. 3, p. 113-118, May 2005.

NAHAS, M. V. Atividade física, saúde e qualidade de vida: conceitos e sugestões para um estilo de vida ativo. 3 ed. Londrina: Atual, 2003.
OSÓRIO, C. D. et al. A comparative study of sleep quality in fibromyalgia. Arthritis \& Rheumatism, Hoboken, v. 46, n. 9, p. S112, 2002.

PASSOS, G. S. et al. Tratamento não farmacológico para a insônia crônica. Revista Brasileira de Psiquiatria, São Paulo, v. 29, n. 3, p. 279-282, set. 2007.

REILLY, T.; EDWARDS, B. Altered sleep-wake cycles and physical performance in athletes. Physioliga \& Behavior, United States, v. 90, n. 2-3, p. 274-284, Feb. 2007.

SHAPIRO, C. M.; BACHMAYER, D.

Epidemiological aspects of sleep in general public and hospital outpatient samples. Acta Physiologica Scandinica. Supplementum, England, v. 574, p. 41-43, 1988.

SHERRILL, D. L.; KOTCHOU, K.; QUAN, S. F. Association of physical activity and human sleep disorders. Archives of Internal Medicine, United States, v. 158, n. 17, p. 1894-1898, Sept.1998.

SINGH, N. A.; CLEMENTS, K. M.; FIATARONE, M. A. A randomized controlled trial of the effect of exercise on sleep. Sleep, United States, v. 20, n. 2, p. 95-101, Feb. 1997.

TANAKA, H. et al. Short naps and exercise improve sleep quality and mental health in the elderly. Psychiatry and Clinical Neurosciences, Australia, v. 56, n. 3, p. 233-234, June 2002.

VUORI, I. et al. Epidemiology of exercise effects on sleep. Acta Physiologica Scandinavian. Supplementum, England, v. 574, p. 3-7, 1988.

WEYDAHL, A. Sex differences in the effect of exercise upon sleep. Perceptual and Motor Skills, v. 72, n. 2, p. 455-465, Apr. 1991.

WORLD HEALTH ORGANIZATION. Obesity: Preventing and managing the global epidemic. Geneva: WHO, 1997 (Report of WHO Consultation on Obesity). 